\title{
A Secure and Privacy Preserving Authentication Protocol for Wireless Sensor Networks in Smart City
}

\author{
Qi Xie ( $\square$ qixie68@126.com ) \\ Keheng Li \\ Hangzhou Normal University \\ Xiao Tan \\ Hangzhou Normal University \\ Lidong Han \\ Hangzhou Normal University \\ Wen Tang \\ Hangzhou Normal University \\ Bin $\mathrm{Hu}$ \\ Hangzhou Normal University
}

Hangzhou Normal University https://orcid.org/0000-0002-8808-3124

\section{Research}

Keywords: wireless sensor networks, authentication, Anonymity, smart city

Posted Date: January 18th, 2021

DOl: https://doi.org/10.21203/rs.3.rs-144556/v1

License: (c) (i) This work is licensed under a Creative Commons Attribution 4.0 International License.

Read Full License 


\title{
A Secure and Privacy Preserving Authentication Protocol for Wireless Sensor Networks in Smart City
}

\author{
Qi Xie*, Keheng Li, Xiao Tan, Lidong Han, Wen Tang and Bin Hu \\ Key Laboratory of Cryptography of Zhejiang Province, Hangzhou Normal University, \\ Hangzhou 311121, China \\ * Correspondence to Qi XIE, qixie68@ 126.com; Tel: +86-571-28866775
}

\begin{abstract}
Smart city can improve the efficiency of managing assets and resources, optimize urban services, and improve the quality of citizens' life. Wireless sensor networks (WSN) can solve many problems in smart city, such as smart transportation, smart healthcare, smart energy, and so on. However, security and privacy are the biggest challenges for WSN. Recently, Banerjee et al. proposed a security enhanced authentication and key agreement scheme for WSN, but their scheme can't resist off-line password guessing attack, impersonation attack, and does not achieve session key secrecy, identity unlinkability and perfect forward secrecy. In order to fix these flaws, a secure and privacy preserving authentication protocol for WSN in smart city is proposed. We prove the security of the proposed protocol by using applied pi calculus based formal verification tool ProVerif, and show that it has high computational efficiency by comparison with some related schemes.
\end{abstract}

Keywords: wireless sensor networks; authentication; Anonymity; smart city

\section{Introduction}

Smart city means to make use of information and communication technology, such as artificial intelligence, Internet of Things and cloud computing etc, to sense, analyze and integrate the key information of urban operation core system, so as to make intelligent response to various needs including people's livelihood, environmental protection, public safety, urban services, industrial and commercial activities, to create a better city life for mankind, build sustainable communities. Wireless sensor networks (WSN) are widely used in smart city, such as environmental monitoring, health care, smart grids and surveillance [1-4]. Through Internet of Things devices, users can access any sensor node in WSN. Therefore, the security of wireless sensor networks is getting more and more attention. Authentication is the first step to ensure the correct transmission of information and the security of WSN. A legitimate user can access a legitimate sensor with user anonymity and receive information from the sensors. Therefore, security and privacy are the biggest challenges for WSN.

In 2004, Watro et al. [5] proposed an authentication protocol for wireless sensor networks based on public key encryption. In order to strengthen the security of the protocol, Das [6] proposed a two-factor authentication protocol using password and smartcard. Khan and Alghathbar [7] proposed a protocol with better performance than Das's protocol. However, the password update phase in their scheme is faulty. Later, Yeh et al. [8] proposed a mutual authentication scheme based on Elliptical Curve Cryptosystem but it has a higher computation cost. Xue et al. [9] proposed a temporal-credential-based protocol in WSN that provides strong authentication and the session among the parties. However, their scheme can't resist many attacks, such as stolen smart card attack, impersonation attack and so on. Later, Jiang et al. [10] proposed an secure three-factor protocol for WSN. Recently, Turkanovic et al. [11] proposed an authentication and key agreement scheme for wireless sensor networks, but Banerjee et al. [12] found that Turkanvic et al.'s scheme can't resist identity theft attack and eavesdropping attack, and then Banerjee et al. proposed an improved scheme based on the biometric and smart card.

Banerjee et al. [12] claimed that their scheme can resist various attacks. However, in this paper, we find that their scheme has some weaknesses, it can't resist off-line password guessing attack and impersonation attack, and does not achieve session key secrecy, identity unlinkability and perfect 
forward secrecy. Therefore, We propose a new scheme to overcome the weaknesses of Banerjee et al.'s scheme.

The rest of the paper is structured as follows: Sections 2 and 3 introduce methods and preliminaries. Sections 4 and 5 review the Banerjee et al.'s scheme and present the attacks on their scheme. The proposed scheme, security analysis, results and discussion are given in Sections 6, 7 and 8. Section 9 is conclusions.

\section{Methods}

The authentication model for WSN consists of users, sensor nodes and gateway nodes. Sensor nodes collect data from their environment, and users can access and receive data from sensor nodes. Gateway nodes are responsible for authentication between users and sensor nodes. In order to prevent unauthorized users from accessing data stored in sensors nodes, before users access sensor nodes, users and sensors nodes should authenticated each other with the help of gateway nodes, and establish session keys to encrypt data transmitted between users and sensors nodes.

The threat assumptions of this model are as follows[13]:

- The adversary can be a user, any registered user can act as an adversary.

- The adversary can intercept or eavesdrop on all communication messages in a public channel, thereby capturing any exchanged messages between a user and gateway or sensor.

- The adversary has the ability to eavesdrop, intercept, modify, or delete the transmitted message.

- The adversary has the ability to obtain all information stored in users' smart cards by using the side channel attack [14].

- An external adversary can also register, login and receive his smart card.

According to above threat assumptions, the proposed protocol for WSN should meet the following security and privacy criteria:

- Mutual authentication and key agreement: user and sensor node should authenticated each other with the help of Gateway node, and establish session key.

- Anonymity and unlinkability: the protocol protects the user's real identity and the adversary can't trace the user's activities.

- Password friendly: the user can update and change his/her password freely.

- No password guessing attacks: the protocol can protect the user's password from guessing attack and ensure the adversary can't verify whether the password is right or not.

- No smart stolen/lost attacks: even if the smart card is lost or stolen, the adversary can obtain all information stored in it, but the adversary can't attack the protocol successfully.

- Perfect forward secrecy: even if an adversary can compromise long secret keys, he or she still can not compute the session keys.

- Known session key security: even if an adversary knows session key, the protocol still safety.

- No replay attack: the protocol prevents the adversary from replaying the transmission information to attack the protocol successfully.

- No various known attacks: the protocol can resist various known attacks, such as forgery attacks, impersonation attacks and man-in-the-middle attacks, etc.

\section{Preliminaries}

In this section, we introduce the elliptic curve cryptosystem, the fuzzy extractor, and some notations which will be used in our protocol.

\subsection{Elliptic Curve Cryptosystem}

The Elliptic Curve Cryptosystem (ECC) is widely used to design password-based authentication protocols, which are created by Miller [15] and Neal [16], respectively. ECC uses the following formula:

$$
y^{2}=x^{3}+a x+b \bmod p, \quad a, b \in F_{p}
$$


The above equation is ECC on $F_{p}$. The following conditions must be met in order to ensure safety:

$$
\begin{array}{r}
4 a^{3}+27 b^{2} \neq 0 \\
644474448
\end{array}
$$

We choose $P$ as a base point on $F_{p}$, then $x P=P+\mathrm{L}+P, x y P$ is a Diffie-Hellman value based on ECC.

\subsection{Fuzzy extractor}

It is very difficult for users to lose and steal their biological information. In many protocols, the user's biometric will be taken as an important factor. There is a slight difference in each extraction of biological information, which can be corrected by using fuzzy extraction. The fuzzy extractor consists of two procedures (Gen, Rep) [17, 18]:

$$
\begin{aligned}
& (\alpha, \beta)=\operatorname{Gen}(B) \\
& \alpha=\operatorname{Rep}\left(B^{*}, \beta\right)
\end{aligned}
$$

Where $B$ is the biometric and $B^{*}$ is closed to $B$. Gen function return a string $\alpha \in\{0,1\}^{k}$ and a coadjutant string $\beta \in\{0,1\}^{*}$. For each biometric $B$, Gen function outputs a key $\alpha$ and a help data $\beta$. For each biometric $B^{*}, \operatorname{Rep}$ function recovers a key $\alpha$ with the help data $\beta$.

\subsection{Notations}

The Notations used in the paper are showed in Table 1.

Table 1 Notations

\begin{tabular}{ll}
\hline Notations & \multicolumn{1}{c}{ Descriptions } \\
\hline$U_{i}$ & i-th user \\
$U S C$ & The user's smart card \\
$S_{j}$ & j-th sensor node \\
$G W N$ & Gateway node \\
$S_{g}$ & Secret key of the gateway node \\
$P K_{g}$ & Public key of the gateway node \\
$G W N P S_{j}$ & Secret key of the gateway node shared with the sensor node \\
$G W N P U_{i}$ & Secret key of the gateway node shared with the user \\
$U I D_{i}$ & User's identity \\
$U P W D_{i}$ & User's password \\
$S I D_{j}$ & Sensor node's identity \\
$B I O_{i}$ & User's biological information \\
$T_{x}$ & Current timestamp \\
$\Delta T$ & Allowed transmission delay \\
$S K$ & Shared session key \\
$E_{x}() / D_{x}()$ & Encryption or decryption function using $x$ \\
$h()$ & One-way hash function \\
$B H()$ & Bio-hash operation \\
$\oplus$ & Performing XOR operation \\
$\|$ &
\end{tabular}

\section{Brife review of Banerjee et al.'s Scheme}


The Banerjee et al.'s scheme [12] has six phases: pre-deployment phase, registration phase, login phase, authentication and key agreement phase, password change phase and dynamic node addition phase. We omit the last two phases.

\subsection{Pre-deployment}

In this phase, the administrator uses the setup server to establish the environment. The setup server chooses identity $S I D_{j}$ for each sensor node $S_{j}$, and provides a key $G W N P S_{j}$ shared with the gateway node $G W N$.The $G W N$ is also provided with a secret key $S_{g}$, and stores $\left\{S I D_{j}, G W N P S_{j}, S_{g}\right\}$.

\subsection{Registration phase}

\subsubsection{User registration phase}

The user $U_{i}$ chooses his identity $U I D_{i}$, password $U P W D_{i}$ and a random number $r_{i}$, and then calculates $M I D_{i}=h\left(U I D_{i} \| r_{i}\right)$ and $M P W D_{i}=h\left(U P W D_{i} \| r_{i}\right) . U_{i}$ sends $\left\{M I D_{i}, M P W D_{i}\right\}$ to the gateway node $G W N$ through secure channel.

After receiving $\left\{M I D_{i}, M P W D_{i}\right\}, G W N$ selects secret key $G W N P U_{i}$ and calculates $M X I P_{i}=h\left(M I D_{i} \| M P W D_{i}\right) \oplus G W N P U_{i}$ and $X_{i}=h\left(M I D_{i} \| S_{g}\right)$, and stores $\left\{M X I P_{i}, X_{i}, h()\right\}$ into the smart card USC and issues it to $U_{i}$ securely.

After receiving $U S C, U_{i}$ calculates $G W N P U_{i}=h\left(M I D_{i} \| M P W D_{i}\right) \oplus M X I P_{i}$, $i m g_{x}=B H\left(r_{i} \oplus B I O_{i}\right), V_{i}=h\left(G W N P U_{i} \| i m g_{x}\right)$ and $A_{i}=h\left(U I D_{i} \| U P W D_{i}\right) \oplus r_{i}$. The user appends $\left\{B H(), V_{i}, A_{i}\right\}$ to the $U S C$.

\subsubsection{Sensors registration phase}

The sensor node $S_{j}$ chooses a random number $r_{j} . S_{j}$ calculates $M X_{j}=h\left(S I D_{j}\left\|r_{j}\right\| G W N P S_{j}\right)$ and $M Y_{j}=r_{j} \oplus G W N P S_{j} . \quad S_{j}$ sends $\quad\left\{S I D_{j}, M X_{j}, M Y_{j}\right\}$ to $G W N$ through secure channel.

After receiving $\left\{S I D_{j}, M X_{j}, M Y_{j}\right\}, \quad G W N \quad$ calculates $\quad r_{j}=M Y_{j} \oplus G W N P S_{j}$ and verifies $M X_{j}=h\left(S I D_{j}\left\|r_{j}\right\| G W N P S_{j}\right)$. And then calculates $P_{j}=h\left(M X_{j} \| S_{g}\right)$. The $G W N$ stores $P_{j}$ securely in its memory and issues $P_{j}$ to $S_{j} . S_{j}$ stores $P_{j}$ securely.

\subsection{Login phase}

The user $U_{i}$ inputs $U I D_{i}, U P W D_{i}$ and $B I O_{i}$. USC calculates $\mathrm{r}_{\mathrm{i}}=\mathrm{h}\left(\mathrm{UID}_{\mathrm{i}} \| \mathrm{UPWD}_{\mathrm{i}}\right) \oplus \mathrm{A}_{\mathrm{i}}$, $i m g_{x}=B H\left(r_{i} \oplus B I O_{i}\right) \quad, \quad M I D_{i}=h\left(U I D_{i} \| r_{i}\right) \quad, \quad M P W D_{i}=h\left(U P W D_{i} \| r_{i}\right) \quad$ and $G W N P U_{i}=h\left(M I D_{i} \| M P W D_{i}\right) \oplus M X I P_{i}$. And then USC verifies $V_{i}$ ? $=h\left(G W N P U_{i} \| i m g_{x}\right)$. If not, the user $U_{i}$ re-does it. Otherwise, the smart card USC chooses a random number $r_{1}$ and calculates $M_{1}=h\left(X_{i}\left\|G W N P U_{i}\right\| r_{1}\right)$ and $M_{2}=r_{1} \oplus X_{i}$. $U_{i}$ sends the request message $\left\{M I D_{i}, M_{1}, M_{2}\right\}$ to sensor node $S_{j}$.

\subsection{Authentication and key agreement phase}

After receiving $\left\{M I D_{i}, M_{1}, M_{2}\right\}, S_{j}$ chooses a random number $r_{2}$ and calculates $M_{3}=P_{j} \oplus r_{2}$ and $M_{4}=h\left(G W N P S_{j}\left\|M_{2}\right\| r_{2}\right) . S_{j}$ sends $\left\{S I D_{j}, M I D_{i}, M_{1}, M_{2}, M_{3}, M_{4}\right\}$ to $G W N$.

After receiving $\left\{S I D_{j}, M I D_{i}, M_{1}, M_{2}, M_{3}, M_{4}\right\} \quad, G W N \quad$ calculates $\quad X_{i}^{*}=h\left(M I D_{i} \| S_{g}\right)$, $r_{1}^{*}=M_{2} \oplus X_{i} \quad, \quad$ and $\quad r_{2}^{*}=M_{3} \oplus P_{j} . \quad G W N \quad$ verifies $\quad M_{1} ?=h\left(X_{i}\left\|G W N P U_{i}\right\| r_{1}^{*}\right) \quad$ and $M_{4}=h\left(G W N P S_{j}\left\|M_{2}\right\| r_{2}^{*}\right)$. If both are equal, the $G W N$ authenticates the user $U_{i}$ and the sensor node $S_{j}$ The $G W N$ chooses a random number $r_{3}$ and calculates $M_{5}=r_{3} \oplus h\left(G W N P S_{j} \oplus r_{2}^{*}\right)$, 
$M_{6}=h\left(X_{i}^{*}\left\|G W N P S_{j}\right\| r_{1}^{*}\left\|r_{2}^{*}\right\| r_{3}\right), \quad P_{1}=r_{1} \oplus P_{j}$ and $P_{2}=X_{i}^{*} \oplus h\left(P_{j}\left\|r_{2}^{*}\right\| r_{1}^{*}\right)$. And then sends $\left\{M_{5}, M_{6}, P_{1}, P_{2}\right\}$ to $S_{j}$.

After receiving $\left\{M_{5}, M_{6}, P_{1}, P_{2}\right\}, S_{j}$ calculates $r_{1}^{*}=P_{1} \oplus P_{j}, \quad X_{i}^{*}=P_{2} \oplus h\left(P_{j}\left\|r_{2}\right\| r_{1}^{*}\right)$, $r_{3}^{*}=M_{5} \oplus h\left(G W N P S_{j} \oplus r_{2}\right)$ and verifies $M_{6} ?=h\left(X_{i}^{*}\left\|G W N P S_{j}\right\| r_{1}^{*}\left\|r_{2}\right\| r_{3}^{*}\right)$. If it is equal, the $S_{j}$ authenticates the $G W N$ and then calculates the session key $S K=h\left(X_{i}^{*}\left\|P_{j}\right\| r_{3}^{*}\left\|r_{2}\right\| r_{1}^{*}\right)$. $S_{j}$ calculate $M_{7}=X_{i}^{*} \oplus r_{2}, M_{8}=\left(P_{j} \| r_{3}^{*}\right) \oplus r_{2}$ and $M_{9}=h\left(r_{1}^{*} \| r_{2}\right) . S_{j}$ sends $\left\{M_{7}, M_{8}, M_{9}\right\}$ to $U_{i}$

After receiving $\left\{M_{7}, M_{8}, M_{9}\right\}, U_{i}$ calculates $r_{2}^{*}=X_{i}^{*} \oplus M_{7},\left(P_{j} \| r_{3}^{*}\right)=M_{8} \oplus r_{2}^{*}$ and then verifies $M_{9} ?=h\left(r_{1} \| r_{2}^{*}\right)$. If the result is equal, $U_{i}$ authenticates $S_{j}$ and then calculates the session key $S K=h\left(X_{i}\left\|P_{j}\right\| r_{3}^{*}\left\|r_{2}^{*}\right\| r_{1}\right)$.

\section{Security Flaws of Banerjee et al.'s Scheme}

Though Banerjee et al. claimed that their scheme can resist various attacks, in this section, we show that their scheme has some security flaws.

\subsection{Identity linkability}

In Banerjee et al.'s scheme, because $M I D_{i}=h\left(U I D_{i} \| r_{i}\right)$ transmitted in public channel and unchanged in each session, so the scheme exists the user identity linkability. Further, the adversary may get the user's real identity according to the user's behavior information. That is, the user's anonymity maybe broken.

\subsection{Off-line password guessing attacks}

If an adversary can obtain $\left\{M X I P_{i}, X_{i}, h(), B H(), V_{i}, A_{i}\right\}$ stored in user's smart card, and $\left\{M I D_{i}, M_{1}, M_{2}\right\}$ from the public channel, then he can guess the user's password UPWD, and computes $\mathrm{r}_{\mathrm{i}}{ }^{\prime}=\mathrm{h}\left(\mathrm{UID}_{\mathrm{i}} \| \mathrm{UPWD}\right) \oplus \mathrm{A}_{\mathrm{i}} \quad, \quad M I D_{i}{ }^{\prime}=h\left(U I D_{i} \| r_{i}{ }^{\prime}\right) \quad, \quad M P W D_{i}{ }^{\prime}=h\left(U P W D \| r_{i}{ }^{\prime}\right) \quad$, $G W N P U_{i}{ }^{\prime}=h\left(M I D_{i}{ }^{\prime} \| M P W D_{i}{ }^{\prime}\right) \oplus M X I P_{i} \quad, \quad r_{1}{ }^{\prime}=M_{2} \oplus X_{i} \quad$ and verifies whether $M_{1}=h\left(X_{i}\left\|G W N P U_{i}{ }^{\prime}\right\| r_{1}{ }^{\prime}\right)$ or not. If yes, the guessed password is correct. Otherwise, the adversary does it again till to find the correct password.

This attack may success, the reason is that the user's identity $U I D_{i}$ may easily to be known (e.g. an insider attacker, like the user's colleague) or it is often publicly available, and the password dictionary size is very restricted. Even if the adversary needs to guess $U I D_{i}$ and $U P W D_{i}$ simultaneously, the time complexity of the above attacking procedure is $\mathrm{O}\left(\left|D_{i d}\right|^{*}\left|D_{p w}\right| *\left(T_{h}\right)\right)$, where $T_{h}$ is the running time for hash operation, and can guess the correct identity and password quickly [19].

If an adversary can get the user's password by offline password guessing attack, then he can know $G W N P U_{i}$ and $X_{i}$, and he can launch impersonation attack.

\subsection{No perfect forward secrecy}

Because the session key is $S K=h\left(X_{i}\left\|P_{j}\right\| r_{3}\left\|r_{2}\right\| r_{1}\right)$, if an adversary can obtain the secret key $S_{g}$ of $G W N$, then he can compute $X_{i}=h\left(M I D_{i} \| S_{g}\right), \quad r_{1}=M_{2} \oplus X_{i}, \quad P_{j}=r_{1} \oplus P_{1}, \quad r_{2}=X_{i} \oplus M_{7}$, $\left(P_{j} \| r_{3}\right)=M_{8} \oplus r_{2}$.That is, the adversary can compute the session key, since he can get $\left\{M I D_{i}, M_{2}, P_{1}, M_{7}, M_{8}\right\}$ from public channels

\subsection{No session key secrecy}

If a legal user $U_{l}$ have pass through the authentication of the sensor node $S_{j}$, then $U_{l}$ can know $P_{j}$. After that, when other user $U_{i}$ wants to pass through the authentication of the sensor node 
$S_{j}, \quad U_{l}$ can get the authentication messages $\left\{M I D_{i}, M_{2}, P_{1}, M_{7}, M_{8}\right\}$ from public channels, so $U_{l}$ can compute $r_{1}=P_{j} \oplus P_{1}, \quad X_{i}=M_{2} \oplus r_{1}, r_{2}=X_{i} \oplus M_{7},\left(P_{j} \| r_{3}\right)=M_{8} \oplus r_{2}$, therefore, $U_{l}$ can compute the session key $S K=h\left(X_{i}\left\|P_{j}\right\| r_{3}\left\|r_{2}\right\| r_{1}\right)$ shared between the $U_{i}$ and $S_{j}$.

\subsection{Impersonation attack}

If a legal user $U_{l}$ have pass through the authentication of the sensor node $S_{j}$, and obtains $P_{j}$, then he can impersonate the $S_{j}$. When other user $U_{i}$ wants to login onto the sensor node $S_{j}, U_{l}$ sends $\left\{M I D_{i}, M_{1}, M_{2}\right\}$ to $S_{j}, S_{j}$ sends $\left\{S I D_{j}, M I D_{i}, M_{1}, M_{2}, M_{3}, M_{4}\right\}$ to GWN. When GWN responses the message $\left\{M_{5}, M_{6}, P_{1}, P_{2}\right\}, U_{l}$ intercepts it, and chooses a random number $r_{3}{ }^{\prime}$, and computes $r_{1}^{*}=P_{1} \oplus P_{j}, r_{2}^{*}=M_{3} \oplus P_{j}, \quad X_{i}^{*}=P_{2} \oplus h\left(P_{j}\left\|r_{2}\right\| r_{1}^{*}\right)$, the session key $S K=h\left(X_{i}^{*}\left\|P_{j}\right\| r_{3}{ }^{\prime}\left\|r_{2}\right\| r_{1}^{*}\right)$, $S_{j}$ calculate $M_{7}=X_{i}^{*} \oplus r_{2}{ }^{*}, M_{8}=\left(P_{j} \| r_{3}{ }^{\prime}\right) \oplus r_{2}{ }^{*}$ and $M_{9}=h\left(r_{1}^{*} \| r_{2}^{*}\right) . U_{l}$ sends $\left\{M_{7}, M_{8}, M_{9}\right\}$ to $U_{l}$. Obviously, $U_{l}$ can compute and verify the correction of the session key $S K$. However, $U_{l}$ shares the session key $S K$ with $U_{l}$, not the sensor node $S_{j}$.

\section{Proposed Scheme}

There are three entities of our proposed scheme: the user $U_{i}$, the sensor node $S_{j}$ and the gateway node $G W N$. The user and the sensor node can authenticate each other and establish a session key with the help of the gateway node. Our protocol has four phases: initialization phase, registration phase, authentication and key agreement phase and password change phase.

\subsection{Initialization phase}

The administrator provides an identity $S I D_{j}$ and a secret key $G W N P S_{j}$ (shared with $G W N$ ) for the sensor node $S_{j}$, and chooses a prime number $P$ and an additive group $G 1$, the $G W N$ 's long secret key $S_{g} \in Z_{p}$ and computes the $G W N$ 's public key $P K_{g}=S_{g} P$ where $P \in G 1$.

\subsection{Registration phase}

The registration phase is run through the secure channel as shown in Algorithm 1. The user $U_{i}$ firstly chooses its identity $U I D_{i}$ and password $U P W D_{i}$ and sends $\left\{U I D_{i}\right\}$ to $G W N$. After receiving $\left\{U I D_{i}\right\}, G W N$ calculates $X_{i}=h\left(U I D_{i} \| S_{g}\right)$. GWN enters $\left\{X_{i}\right\}$ into the smart card and sends it to $U_{i}$ through the secure channel.

$U_{i}$ imprints the biological information $B I O_{i}$ and calculates $(\alpha, \beta)=\operatorname{Gen}\left(B I O_{i}\right)$, $V_{1}=h\left(U I D_{i}\left\|U P W D_{i}\right\| \alpha\right)$ and $Y_{i}=X_{i} \oplus h\left(U I D_{i} \| \alpha\right) . U_{i}$ stores $\left\{Y_{i}, \operatorname{Rep}(), V_{1}, \beta\right\}$ in the smart card USC.

\begin{tabular}{|cc|}
\hline$U_{i}$ & $G W N$ \\
$\left(U I D_{i}, U P W D_{i}, B I O_{i}\right)$ & \\
\hline$U I D_{i}$ & \\
& \\
& \\
$(\alpha, \beta)=G e n\left(S_{i}\right)$ & \\
$V_{1}=h\left(U I D_{i} \| S_{g}\right)$ \\
$Y_{i}=X_{i} \oplus h\left(U I D_{i}\right)$ \\
$U S C:\left\{Y_{i}, \operatorname{Rep}(), V_{1}, \beta\right\}$
\end{tabular}

Algorithm 1: User-gateway registration phase 


\subsection{Authentication and key agreement phase}

The user, the sensor and the gateway authenticate with each other, and the user and the sensor negotiate session key as shown in Algorithm 2.

Step 1. $U_{i}$ inserts smart card and inputs identity $U I D_{i}$, password $U P W D_{i}$ and biological information $B I O_{i}$. The smart card USC calculates $\alpha=\operatorname{Rep}\left(B I O_{i}, \beta\right)$ and verifies whether $V_{1} ?=h\left(U I D_{i}\left\|U P W D_{i}\right\| \alpha\right)$ or not. If not, the user re-does it. Otherwise, USC chooses a random numbers $a$ and calculates the user's temporary identity $P I D_{i}=U I D_{i} \oplus h\left(a P K_{g}\right), T_{u}=a P$, $X_{i}=Y_{i} \oplus h\left(U I D_{i} \| \alpha\right)$, and $M_{1}=h\left(U I D_{i}\left\|X_{i}\right\| T_{u} \| T_{1}\right)$ where $T_{1}$ is the current timestamp. $U_{i}$ sends $\left\{P I D_{i}, S I D_{j}, M_{1}, T_{u}, T_{1}\right\}$ to $G W N$.

Step 2. After receiving $\left\{P I D_{i}, S I D_{j}, M_{1}, T_{u}, T_{1}\right\}, G W N$ checks the validity of $T_{1}$ and $S I D_{j}$, and forwards $\left\{S I D_{j}\right\}$ to the sensor node $S_{j}$.

Step 3. After receiving $\left\{S I D_{j}\right\}, S_{j}$ chooses a random number $b$ and calculates $T_{s}=b P$, $M_{2}=T_{s} \oplus h\left(G W N P S_{j} \| T_{2}\right) \quad$ and $\quad M_{3}=h\left(S I D_{j}\left\|G W N P S_{j}\right\| T_{s} \| T_{2}\right)$ where $T_{2}$ is the current timestamp. $S_{j}$ sends $\left\{S I D_{j}, M_{3}, M_{4}, T_{2}\right\}$ to the $G W N$.

\begin{tabular}{|c|c|c|}
\hline $\begin{array}{c}U_{i} \\
\left(U I D_{i}, U P W D_{i}, B I O_{i}\right)\end{array}$ & $\begin{array}{c}G W N \\
\left(S_{g}, P K_{g}\right)\end{array}$ & $\begin{array}{c}S_{j} \\
\left(S I D_{j}, G W N P S_{j}\right)\end{array}$ \\
\hline $\begin{array}{l}\text { Step 1: } \\
\text { Input } U I D_{i}, U P W D_{i}, B I O_{i} \\
\alpha=\operatorname{Rep}\left(B I O_{i}, \beta\right) \\
V_{1} ?=h\left(U I D_{i}\left\|U P W D_{i}\right\| \alpha\right) \\
\text { choose } a \\
P I D_{i}=U I D_{i} \oplus h\left(a P K_{g}\right) \\
T_{u}=a P \\
X_{i}=Y_{i} \oplus h\left(U I D_{i} \| \alpha\right) \\
M_{1}=h\left(U I D_{i}\left\|X_{i}\right\| T_{u} \| T_{1}\right) \\
\quad\left\{P I D_{i}, S I D_{j}, M_{1}, T_{u}, T_{1}\right\} \\
\text { Step } 5: \\
\text { Ver } T_{3} \\
\left(T_{s}, U I D_{i}, S I D_{j}, T_{3}\right)=D_{X_{i}}\left(M_{6}\right) \\
M_{7} ?=h\left(X_{i}\left\|T_{s}\right\| S I D_{j}\left\|U I D_{i}\right\| T_{3}\right. \\
S K=h\left(a T_{s}\left\|U I D_{i}\right\| S I D_{j} \| T_{3}\right) \\
V_{u}=h\left(S K \| T_{5}\right) \\
\quad\left\{V_{u}, T_{5}\right\} \\
\\
\text { Step 6: } S_{j} \\
V_{s} ?=h\left(S K \| T_{4}\right)\end{array}$ & $\begin{array}{l}\text { Step 4: } \\
\text { Ver } T_{1}, T_{2} \\
U I D_{i}=P I D_{i} \oplus h\left(S_{g} T_{u}\right) \\
X_{i}=h\left(U I D_{i} \| S_{g}\right) \\
M_{1} ?=h\left(U I D_{i}\left\|X_{i}\right\| T_{u} \| T_{1}\right) \\
T_{s}=M_{2} \oplus h\left(G W N P S_{j} \| T_{2}\right) \\
M_{3} ?=h\left(S_{j} D_{j}\left\|G W N P S_{j}\right\| T_{s} \| T_{2}\right) \\
M_{4}=E_{G W N P S_{j}}\left(T_{u}, U I D_{i}, S I D_{j}, T_{3}\right) \\
M_{5}=h\left(G W N P S_{j}\left\|T_{u}\right\| U I D_{i}\left\|S I D_{j}\right\| T_{3}\right. \\
M_{6}=E_{X_{i}}\left(T_{s}, U I D_{i}, S I D_{j}, T_{3}\right) \\
M_{7}=h\left(X_{i}\left\|T_{s}\right\| S I D_{j}\left\|U I D_{i}\right\| T_{3}\right) \\
\stackrel{\left\{M_{4}, M_{5}, T_{3}\right\}}{\longrightarrow} S_{j} \\
U_{i} \stackrel{\left\{M_{6}, M_{7}, T_{3}\right\}}{\longleftarrow}\end{array}$ & $\begin{array}{l}\text { Step 3: } \\
\text { choose } b \\
T_{s}=b P \\
M_{2}=T_{s} \oplus h\left(G W N P S_{j} \| T_{2}\right) \\
M_{3}=h\left(S I D_{j}\left\|G W N P S_{j}\right\| T_{s} \| T_{2}\right) \\
G W N \stackrel{\left\{S I D_{j}, M_{2}, M_{3}, T_{2}\right\}}{\longleftarrow} \\
\text { Step 5: } \\
\text { Ver } T_{3} \\
\left(T_{u}, U I D_{i}, S I D_{j}, T_{3}\right)=D_{G W N P S_{j}}\left(M_{4}\right) \\
M_{5} ?=h\left(G W N P S_{j}\left\|T_{u}\right\| U I D_{i}\left\|S I D_{j}\right\| T_{3}\right. \\
S K=h\left(b T_{s}\left\|U I D_{i}\right\| S I D_{j} \| T_{3}\right) \\
V_{5}=h\left(S K \| T_{4}\right) \\
U_{i} \stackrel{\left\{V_{5}, T_{4}\right\}}{ } \\
\text { Step 6: } \\
V_{u} ?=h\left(S K \| T_{5}\right)\end{array}$ \\
\hline
\end{tabular}

Algorithm 2: Authentication and key agreement phase

Step 4. After received the authentication message, $G W N$ first verifies the timestamp $T_{2}$. If $T-T_{2} \leq \Delta T$ is false where $T$ is the current timestamp, GWN refuses the authentication request. Otherwise, $\quad G W N \quad$ calculates $\quad U I D_{i}=P I D_{i} \oplus h\left(S_{g} T_{u}\right) \quad, \quad X_{i}=h\left(U I D_{i} \| S_{g}\right) \quad$ and verifies 
$M_{1} ?=h\left(U I D_{i}\left\|X_{i}\right\| T_{u}\left\|T_{g}\right\| T_{1}\right)$. If the result is false, GWN terminates the protocol. Otherwise, $G W N$ authenticates the user successfully. After that, $G W N$ calculates $T_{s}=M_{2} \oplus h\left(G W N P S_{j} \| T_{2}\right)$ and verifies $M_{3} ?=h\left(S I D_{j}\left\|G W N P S_{j}\right\| T_{s} \| T_{2}\right)$. If the result is false, GWN also terminates the protocol. Otherwise, GWN authenticates the sensor node $S_{j}$ successfully. Then, GWN computes $M_{4}=E_{G W N P S_{j}}\left(T_{u}, U I D_{i}, S I D_{j}, T_{3}\right) \quad$ and $\quad M_{6}=E_{X_{i}}\left(T_{s}, U I D_{i}, S I D_{j}, T_{3}\right) \quad$ and calculates $M_{5}=h\left(G W N P S_{j}\left\|T_{u}\right\| U I D_{i}\left\|S I D_{j}\right\| T_{3}\right)$ and $M_{7}=h\left(X_{i}\left\|T_{s}\right\| S I D_{j}\left\|U I D_{i}\right\| T_{3}\right)$, where $T_{3}$ is the current time stamp. $G W N$ sends $\left\{M_{4}, M_{5}, T_{3}\right\}$ to $S_{j}$ and $\left\{M_{6}, M_{7}, T_{3}\right\}$ to $U_{i}$.

Step 5. After receiving the message $\left\{M_{4}, M_{5}, T_{3}\right\}, S_{j}$ firstly verifies $T_{3}$. If $T^{\prime}-T_{3} \leq \Delta T$ is false where $T^{\prime}$ is the current timestamp, $S_{j}$ terminates the protocol. Otherwise, $S_{j}$ computes $\left(T_{u}, U I D_{i}, S I D_{j}, T_{3}\right)=D_{G_{W N P S}}\left(M_{4}\right)$ and verifies $M_{5} ?=h\left(G W N P S_{j}\left\|T_{u}\right\| U I D_{i}\left\|S I D_{j}\right\| T_{3}\right)$. If the result is equal, $S_{j}$ authenticates $G W N$ successfully. And then, $S_{j}$ calculates the session key $S K=h\left(b T_{u}\left\|U I D_{i}\right\| S I D_{j} \| T_{3}\right)$ and $V_{s}=h\left(S K \| T_{4}\right)$ where $T_{4}$ is the current timestamp. $S_{j}$ sends $\left\{V_{s}, T_{4}\right\}$ to $U_{i}$.

At the same time, when $U_{i}$ receives $\left\{M_{6}, M_{7}, T_{3}\right\}$ from $G W N, U_{i}$ firstly verifies $T_{3}$. If $T^{\prime \prime}-T_{3} \leq \Delta T$ is false where $T^{\prime \prime}$ is the current timestamp, $U_{i}$ terminates the protocol. Otherwise, $U_{i}$ decrypt $\left(T_{s}, U I D_{i}, S I D_{j}, T_{3}\right)=D_{X_{i}}\left(M_{6}\right)$ and verifies $M_{7} ?=h\left(X_{i}\left\|T_{s}\right\| S I D_{j}\left\|U I D_{i}\right\| T_{3}\right)$. If the result is false, $U_{i}$ also terminates the protocol. Otherwise, $U_{i}$ authenticates GWN successfully, and calculates the session key $S K=h\left(a T_{s}\left\|U I D_{i}\right\| S I D_{j} \| T_{3}\right)$ and $V_{u}=h\left(S K \| T_{5}\right)$, where $T_{5}$ is the current timestamp. Then $U_{i}$ sends $\left\{V_{u}, T_{5}\right\}$ to $S_{j}$.

Step 6. After receiving $\left\{V_{s}, T_{4}\right\}$ and $\left\{V_{u}, T_{5}\right\}, U_{i}$ and $S_{j}$ verifies the freshness of $T_{4}$ and $T_{5}$, and verifies the correctness of $V_{s}$ and $V_{u}$, respectively. After verification of correctness, $U_{i}$ and $S_{j}$ share the session key $S K$.

\subsection{Password change phase}

If the user wants to change or update his passwords, $U_{i}$ inserts $U S C$ in card reader and inputs identity $U I D_{i}$, password $U P W D_{i}^{\text {old }}$ and biological information $B I O_{i}$. Next, the smart card calculates $\alpha=\operatorname{Rep}\left(B I O_{i}, \beta\right)$ and verifies $V=h\left(U I D_{i}\left\|U P W D_{i}^{\text {old }}\right\| \alpha\right)$. If the result is false, the smart card doesn't recognize him as a legitimate user. Otherwise, the user inputs new password $U P W D_{i}^{\text {new }}$. The smart card calculates $V^{\text {new }}=h\left(U I D_{i}\left\|U P W D_{i}^{\text {new }}\right\| \alpha\right)$, and replaces $V$ with $V^{\text {new }}$.

\section{Security Analysis}

In the section, we analyze the security of the proposed protocol by using formal and informal security analysis.

\subsection{Formal security analysis}

We simulate our scheme using Proverif simulation tool [20], which is widely used for proving the authentication and session key secrecy. We defined three public channels ch1, ch2, ch3. The details of variables and functions are shown in Table 2. The details of process of user authentication are shown in Table 3. The process of sensor authentication is shown in Table 4. The simulation process of gateway is shown in Table 5.

We use the Proverif to prove our authentication phase. First, the smart card authenticates the user successfully. Second, the gateway node authenticates the user successfully. Third, the sensor node authenticates the gateway node successfully. Fourth, the gateway node authenticates the sensor node successfully. Finally, the user authenticates the gateway node and the sensor node successfully. That is, users, sensors and gateway achieve mutual authentication each other. If the scheme successfully goes on, 
the user and sensor node will negotiate the same session key. Therefore, our scheme has six time points and their code is represented in the Proverif as follows:

event SmartcardAccept(user) means the user logins the smart card successfully.

event gwnAcceptU(user) means the gateway node authenticates the user successfully.

event gwnAcceptS(sensor) means the gateway node authenticates the sensor node successfully.

event sensorAccept $(\mathrm{GWN})$ means the sensor node authenticates the gateway node successfully.

event userAccept(sensor,GWN) means the user authenticates the gateway node and the sensor node successfully.

event Sksuccessful(user,sensor) means user and sensor node get the same session key.

The authentication order of our protocol is as follows:

query Ui:user; inj-event(gwnAcceptU(Ui))==>inj-event(SmartcardAccept(Ui)).

query Ui:user,Gateway:GWN; inj-event(sensorAccept(Gateway)) $\quad \Rightarrow \quad$ inj-event (gwnAcceptU(Ui)).

query Sj:sensor,Gateway:GWN; inj-event(gwnAcceptS(Sj)) $\quad \Rightarrow \quad$ inj-event (sensorAccept(Gateway)).

query Ui:user,Sj:sensor,Gateway:GWN; inj-event(userAccept(Sj,Gateway)) ==> inj-event (gwnAcceptS(Sj)).

Our protocol must also protect the session keys (SKus and SKsu). The code is:

query attacker(SKsu).

query attacker(SKus).

Finally, we use the following code to start the verification:

process

(*constant computed*)

let $\mathrm{Xi}=\mathrm{hash}(\operatorname{concat}(\mathrm{Sg}, \mathrm{UIDi}))$ in

let (ai:bitstring,bi:bitstring) $=$ gen $(\mathrm{BIOi})$ in

let $\mathrm{V} 1=$ hash(concat(UIDi,concat(UPWDi,ai)) ) in

let $\mathrm{Yi}=\mathrm{XOR}(\mathrm{Xi}$,hash(concat(UIDi,ai))) in

!processuser |!processsensornode|!processgateway

The simulation authentication result is shown in Fig. 1.The simulation result of the session key is shown in Fig. 2. The result shows that our scheme achieves mutual authentication and the session key security.

Table 2 Variables and functions

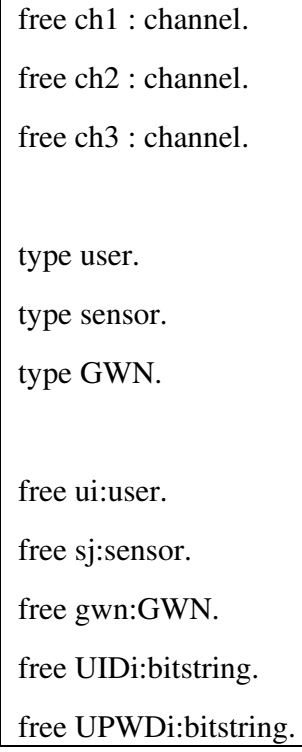




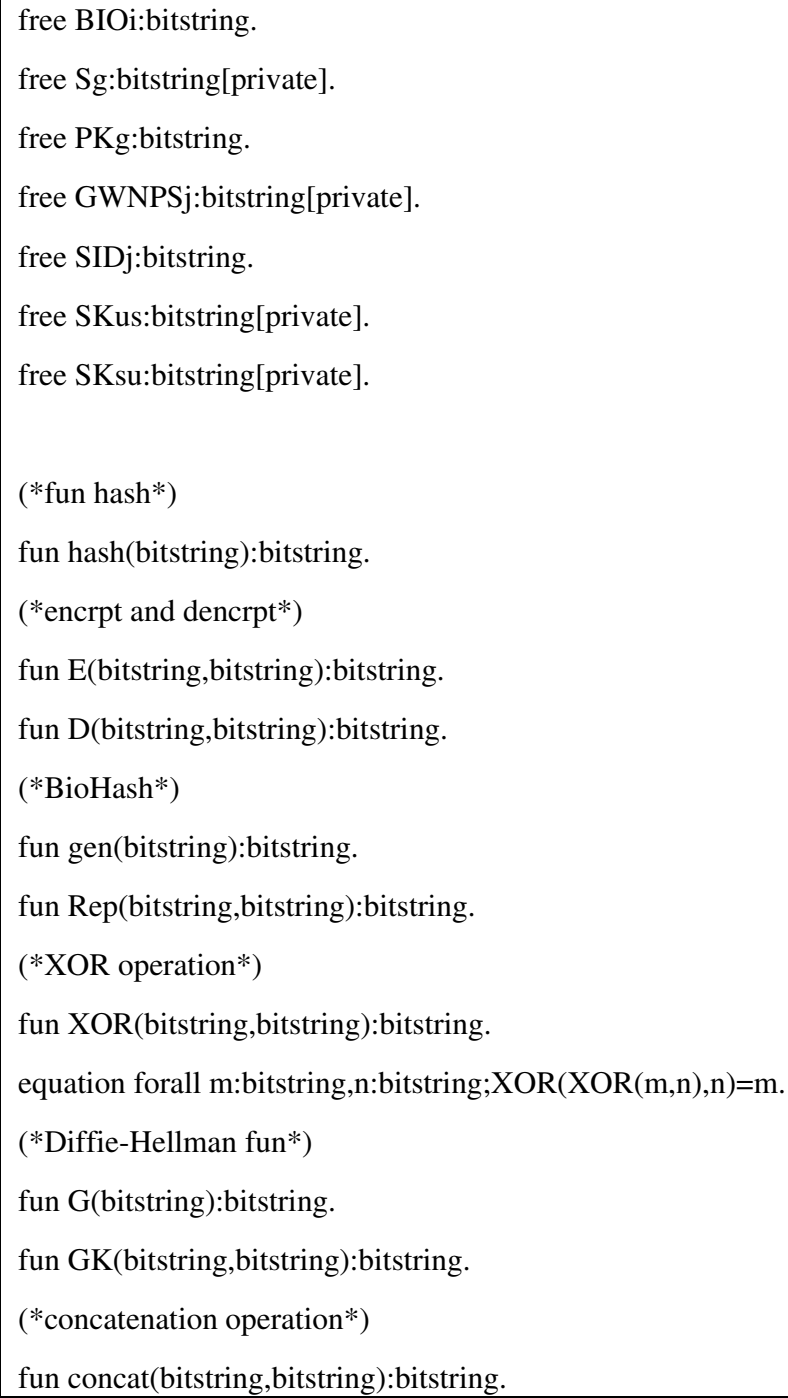

Table 3 User simulation process

\section{(*processuser*)}

let processuser $=$

new bi:bitstring;

new V:bitstring;

let ai=Rep(BIOi,bi) in

let $V^{\prime}=$ hash(concat(concat(UIDi,UPWDi),ai)) in

if $\mathrm{V}^{\prime}=\mathrm{V}$ then

event SmartcardAccept(ui);

new T1:bitstring;

new a:bitstring;

new Yi:bitstring;

let $\mathrm{Tu}=\mathrm{G}(\mathrm{a})$ in

let $\mathrm{Xi}=\mathrm{XOR}(\mathrm{Yi}, \mathrm{hash}($ concat(UIDi,ai)) $)$ in

let $\mathrm{PIDi}=\mathrm{XOR}(\mathrm{UIDi}$,hash $(\mathrm{GK}(\mathrm{a}, \mathrm{PKg})))$ in 
out(ch1,(PIDi,SIDj,M1,Tu,T1));

in(ch1, (M6:bitstring,M7:bitstring,T3:bitstring));

let (Ts:bitstring,UIDi:bitstring,SIDj:bitstring,T3:bitstring $)=\mathrm{D}(\mathrm{Xi}, \mathrm{M} 6)$ in

let M7' $=$ hash $(\operatorname{concat}(\mathrm{Xi}, \operatorname{concat}(\mathrm{Ts}, \operatorname{concat}(\mathrm{SIDj}, \operatorname{concat}(\mathrm{UIDi}, \mathrm{T} 3)))))$ in

let SKus=hash(concat(GK(a,Ts),concat(UIDi,concat(SIDj,T3))))in

new T5:bitstring;

let $\mathrm{Vu}=\mathrm{hash}(\operatorname{concat}(\mathrm{SKus}, \mathrm{T} 5))$ in

out(ch3,(Vu,T5));

in(ch3,(Vs:bitstring,T4:bitstring));

let Vs'=hash(concat(SKus,T4)) in

event Sksuccessful(ui,sj).

Table 4 Sensor node simulation process

(*processsensornode*)

let processsensornode $=$

in(ch2,SIDj:bitstring);

new b:bitstring;

new T2:bitstring;

let $\mathrm{Ts}=\mathrm{G}(\mathrm{b})$ in

let $\mathrm{M} 2=\mathrm{XOR}(\mathrm{Ts}$, hash $(\operatorname{concat}(\mathrm{GWNPSj}, \mathrm{T} 2)))$ in

let M3=hash(concat $($ SIDj, concat $($ GWNPSj,concat $(T s, T 2))))$ in

out(ch2,(SIDj,M2,M3,T2));

in(ch2,(M4:bitstring,M5:bitstring,T3:bitstring));

let (Tu:bitstring,UIDi:bitstring,SIDj:bitstring,T3:bitstring $)=\mathrm{D}(\mathrm{GWNPSj}, \mathrm{M} 4)$ in

let M5'=hash(concat $($ GWNPSj,concat $($ Tu,concat $($ UIDi,concat $(\operatorname{SIDj}, T 3))))$ ) in

if M5=M5' then

event sensorAccept(gwn);

let $\mathrm{SKsu}=\mathrm{hash}(\operatorname{concat}(\mathrm{GK}(\mathrm{b}, \mathrm{Ts})$, concat(UIDi,concat(SIDj,T3))) ) in

new T4:bitstring;

let Vs=hash(concat(SKsu,T4))in

out(ch3,(Vs,T4));

in(ch3,(Vu:bitstring,T5:bitstring));

let $\mathrm{Vu}^{\prime}=$ hash(concat(SKsu,T5))in

if $\mathrm{Vu}^{\prime}=\mathrm{Vu}$ then

event Sksuccessful(ui,sj).

Table 5 Gateway node simulation process

(*processgateway*)

let processgateway=

in(ch1,(SIDj:bitstring,PIDi:bitstring,M1:bitstring,Tu:bitstring,T1:bitstring));

in(ch2,(SIDj:bitstring,M2:bitstring,M3:bitstring,T2:bitstring));

let $\mathrm{UIDi}=\mathrm{XOR}(\mathrm{PIDi}, \mathrm{hash}(\mathrm{GK}(\mathrm{Sg}, \mathrm{Tu})))$ in 
let $\mathrm{Xi}=$ hash(concat(UIDi,Sg)) in

let $\mathrm{M} 1 '=$ hash $(\operatorname{concat}(\mathrm{UIDi}, \operatorname{concat}(\mathrm{Xi}, \operatorname{concat}(\mathrm{Tu}, \mathrm{T} 1))))$ in

if $\mathrm{M} 1{ }^{\prime}=\mathrm{M} 1$ then

event gwnAcceptU(ui);

let $\mathrm{Ts}=\mathrm{XOR}(\mathrm{M} 2$,hash $(\operatorname{concat}(\mathrm{GWNPSj}, \mathrm{T} 2)))$ in

let M3'=hash $(\operatorname{concat}(\mathrm{SIDj}, \operatorname{concat}(\mathrm{GWNPSj}, \operatorname{concat}(\mathrm{Ts}, \mathrm{T} 2))))$ in

if $\mathrm{M} 3{ }^{\prime}=\mathrm{M} 3$ then

event gwnAcceptS(sj);

new T3:bitstring;

let M4=E(GWNPSj,concat(Tu,concat(UIDi,concat(SIDj,T3))))in

let M5=hash(concat(GWNPSj, concat(Tu,concat(UIDi,concat(SIDj,T3)))))in

let M6=E(Xi,concat(Ts,concat(UIDi,concat(SIDj,T3))) in

let $\mathrm{M} 7=$ hash $(\operatorname{concat}(\mathrm{Xi}, \operatorname{concat}(\mathrm{Ts}$, concat(SIDj,concat(UIDi,T3)))))in

out(ch2,(M4,M5,T3));

out(ch1,(M6,M7,T3)).

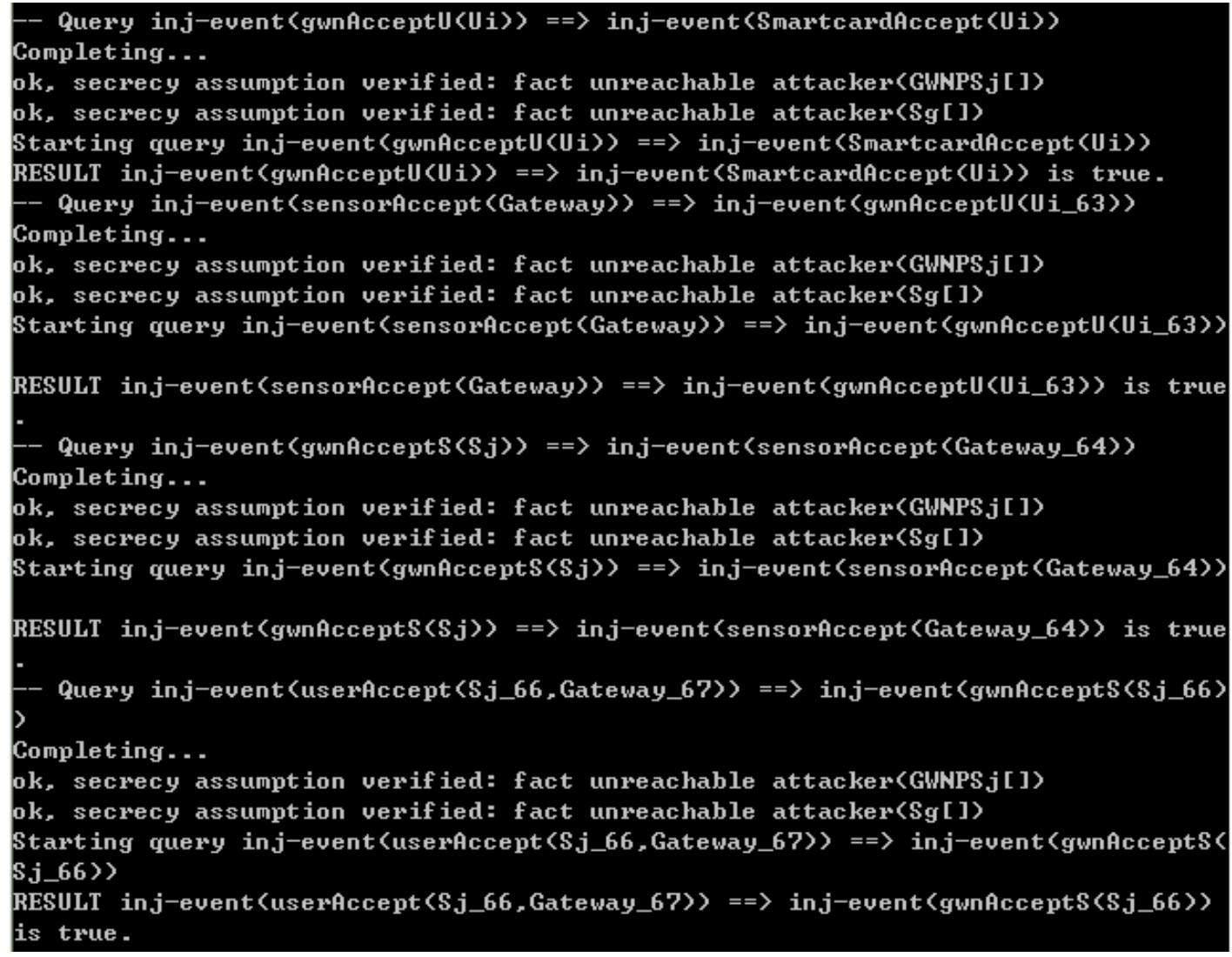

Fig 1: Simulation result of the authentication 


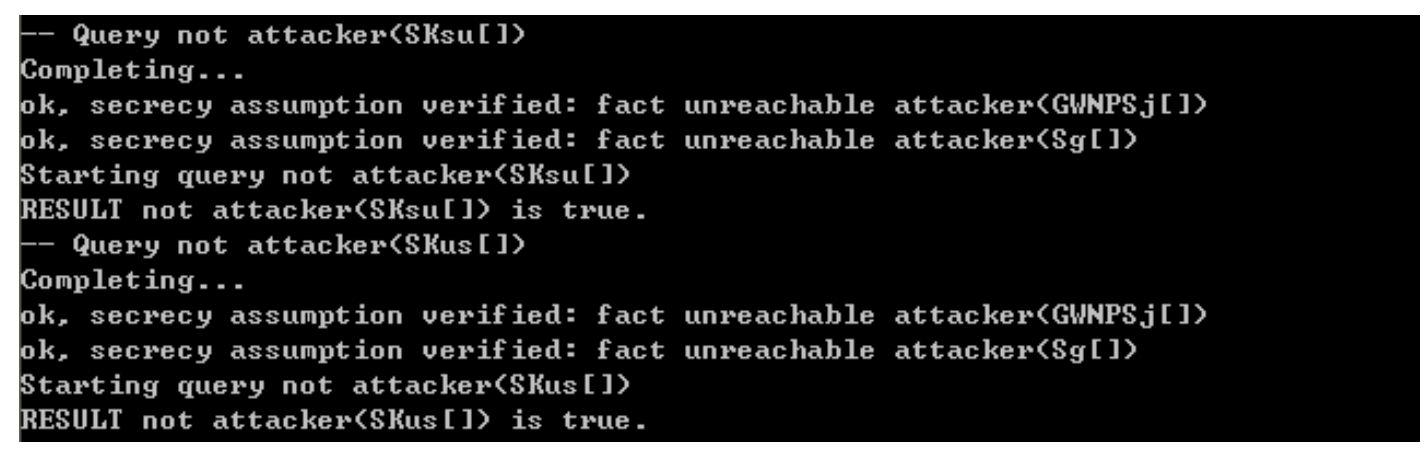

Fig 2: Simulation result of the session key

\subsection{Informal security analysis}

\subsubsection{Anonymity and unlinkability}

In our scheme, the user's real identity is contained in those parameters $<P I D_{i}, M_{4}, M_{5}, M_{6}, M_{7}>$. PID $i$ is protected by Diffie-Hellman problem. And the $M_{4}$ and $M_{6}$ are encrypted by $X_{i}$ and $G W N P S_{j}$ respectively. The rest of the parameters $\left\langle M_{6}, M_{8}\right\rangle$ are protected by the hash function. So, the adversary can't know the user's real identity. Our scheme meets the need of anonymity. The $P I D_{i}$ changes in each session because of the use of the random number and Diffie-Hellman value. So, our scheme is also unlinkability.

\subsubsection{Offline password guessing attacks}

Assume that an adversary knows the parameters $\left\{Y_{i}, \operatorname{Rep}(), V_{1}, \beta\right\}$ stored in the smart card and all messages transmitted in all public channels, but he can't guess the true password. Since the user's password is protected by the bio-information and the hash function, the adversary can't verifier the parameter $V_{1}$. On the other hand, we assume that an adversary knows the legal user's password and all messages transmitted through the public channel, but don't know the parameters stored in the smart card. However, the adversary can't login the protocol because he can't obtain the bio-information. So, our scheme can resist the offline password guessing attacks.

\subsubsection{Replay attack}

There are two ways to prevent replay attacks: adding timestamps and random numbers. Our scheme uses time stamps to prevent replay attack. In every session, the timestamps are different and the entity checks the fresh of the timestamps.

\subsubsection{Impersonation attack and man-in-the-middle attack}

In our scheme, the gateway node authenticates the user by the parameter $M_{1}$. The user authenticates the gateway node by the parameter $M_{7}$. If an adversary wants to impersonate the legal user, he must know those parameter $<T_{u}, X_{i}, T_{s}>$ which $X_{i}$ is pre-shared with the gateway node. However, $X_{i}$ is contained in $Y_{i}$ stored in the smart card securely. Similarly, an adversary can't impersonate the sensor node because of $G W N P S_{j}$. If an adversary captures a sensor node, he cannot know the others' key parameters. So he can't impersonate other sensor node and the user. Therefore, our scheme resists impersonation attack, and the man-in-the-middle is also invalid.

\subsubsection{Perfect forward secrecy}


Assume that an adversary knows the user's password $U P W D_{i}$, the sensor node's secret key $G W N P S_{j}$. Since the session key is $S K=h\left(a b P\left\|U I D_{i}\right\| S I D_{j} \| T_{3}\right)$, an adversary can't compute $a b P$ due to Elliptic Curve Diffie-Hellman problem (ECDHP). So he can't compute the session key.

\subsubsection{Known session key security}

In our scheme, the adversary cannot compute the session key because of ECDHP. The session key is also different in each session due to two random numbers $a, b$. So, if the adversary knows a session key, he cannot know the before and the future session keys.

\section{Results and Discussion}

In this section, we will discuss security and performance comparison with some related schemes, such as Banerjee et al. [12], Wu et al. [1], Choi et al. [21], Park et al. [22], Yeh et al. [8] and Challa et al.'s schemes [23].

$T_{m}$ means the time of the point multiplicative operation in ECC, $T_{R e p}$ means the running time to performance Rep which is equal to $T_{m}$ [24], $T_{s}$ means the time in symmetric encryption or decryption, $T_{h}$ means the time of hash operation, and $T$ is the time or searching the identity in verification table which is related to the number of users. The running time are shown in Table 6 [25].

As shown in Tables 7 an 8 , we can know that the proposed scheme achieves both security and computational efficiency.

Table 6 Nations of time symbols

\begin{tabular}{ccc}
\hline Symbol & Meaning & Time $(\mathbf{m s})$ \\
\hline$T_{m}$ & Time of the point multiplicative operation & 2.226 \\
$T_{R e p}$ & Time of fuzzy extractor & 2.226 \\
$T_{s}$ & Time in symmetric encryption or decryption & 0.0046 \\
$T_{h}$ & Time of hash operation & 0.0023 \\
$T$ & The time for searching the identity in verification table & $O(n), n$ is the \\
& & \\
& & number of users \\
\hline
\end{tabular}

Table 7 Computation cost comparison

\begin{tabular}{llllll}
\hline References & user $(\mathbf{m s})$ & gateway $(\mathbf{m s})$ & Sensor $(\mathbf{m s})$ & Total & Total(ms) \\
\hline$[12]$ & $9 T_{h}+T_{\text {Rep }}$ & $6 T_{h}$ & $6 T_{h}$ & $20 T_{h}+T_{\text {Rep }}$ & $=2.27$ \\
{$[1]$} & $12 T_{h}+T_{\text {Rep }}+2 T_{m}$ & $10 T_{h}$ & $3 T_{h}+2 T_{m}$ & $25 T_{h}+T_{R e p}+4 T_{m}$ & $=11.19$ \\
{$[21]$} & $10 T_{h}+T_{R e p}+2 T_{m}+T_{s}$ & $10 T_{h}+2 T_{s}$ & $6 T_{h}+2 T_{m}+T_{s}$ & $26 T_{h}+T_{R e p}+4 T_{m}+5 T_{s}$ & $=16.19$ \\
{$[22]$} & $10 T_{h}+T_{\text {Rep }}+2 T_{m}$ & $11 T_{h}+T$ & $4 T_{h}+2 T_{m}$ & $25 T_{h}+T_{R e p}+4 T_{m}+T$ & $=11.19+O(n)$ \\
{$[8]$} & $1 T_{h}+2 T_{m}$ & $4 T_{h}+4 T_{m}$ & $3 T_{h}+2 T_{m}$ & $8 T_{h}+8 T_{m}$ & $=17.83$ \\
{$[23]$} & $5 T_{h}+T_{\text {Rep }}+5 T_{m}$ & $4 T_{h}+5 T_{m}+T$ & $3 T_{h}+4 T_{m}$ & $12 T_{h}+T_{\text {Rep }}+14 T_{m}+T$ & $=33.42+O(n)$ \\
Ours & $8 T_{h}+T_{\text {Rep }}+3 T_{m}+T_{s}$ & $7 T_{h}+T_{m}+2 T_{s}$ & $5 T_{h}+2 T_{m}+T_{s}$ & $20 T_{h}+T_{\text {Rep }}+6 T_{m}+4 T_{s}$ & $=15.646$ \\
\hline
\end{tabular}

Table 8 Comparison of security features

\begin{tabular}{lccccccc}
\hline Security features & [12] & [1] & [23] & [21] & [22] & [8] & ours \\
\hline Impersonation attack & $\times$ & $\sqrt{ }$ & $\sqrt{ }$ & $\times$ & $\sqrt{ }$ & $\times$ & $\sqrt{ }$ \\
Anonymity and unlinkability & $\times$ & $\times$ & $\times$ & $\times$ & $\times$ & $\times$ & $\sqrt{ }$ \\
Verification table stolen attack & $\sqrt{ }$ & $\sqrt{ }$ & $\times$ & $\sqrt{ }$ & $\times$ & $\sqrt{ }$ & $\sqrt{ }$ \\
\hline
\end{tabular}




\begin{tabular}{lccccccc}
\hline Password guessing attack & $\times$ & $\times$ & $\sqrt{ }$ & $\sqrt{ }$ & $\sqrt{ }$ & $\times$ & $\sqrt{ }$ \\
Known session key security & $\times$ & $\sqrt{ }$ & $\sqrt{ }$ & $\sqrt{ }$ & $\sqrt{ }$ & $\times$ & $\sqrt{ }$ \\
Replay attack & $\times$ & $\sqrt{ }$ & $\sqrt{ }$ & $\sqrt{ }$ & $\sqrt{ }$ & $\times$ & $\sqrt{ }$ \\
Man-in-the-middle attack & $\times$ & $\sqrt{ }$ & $\sqrt{ }$ & $\sqrt{ }$ & $\sqrt{ }$ & $\times$ & $\sqrt{ }$ \\
Perfect forward secrecy & $\times$ & $\sqrt{ }$ & $\sqrt{ }$ & $\sqrt{ }$ & $\sqrt{ }$ & $\times$ & $\sqrt{ }$ \\
\hline
\end{tabular}

\title{
9. Conclusions
}

In this paper, we have showed that the recently proposed Banerjee et al.'s protocol can't resist off-line password guessing attack, impersonation attack, and does not achieve session key secrecy, identity unlinkability and perfect forward secrecy. Then, we proposed a secure and privacy preserving protocol to fix their security flaws. According to the formal security proof and performance comparison with some related schemes, we can know that our protocol achieves both security and computational efficiency, and can be used to the smart city. In the future, we will design more secure authentication protocols for smart city applications, such as smart transportation and smart healthcare.

Availability of data and materials: The dataset used and analyzed during the current study is available in the manuscript.

\begin{abstract}
Abbreviations:
WSN: Wireless sensor networks

USC: The user's smart card

GWN: Gateway node
\end{abstract}

Conflict of Interests: The authors declare that there is no conflict of interests regarding the publication of this paper.

Funding: This research was supported by the National Key R\&D Program of China (Grant No.2017YFB0802000, URL http://www.most.gov.cn/).

Acknowledgements: This research was supported by the National Natural Science Foundation of China (Grant Nos. 61702152 and 61702153, URL http://www.nsfc.gov.cn/).

\section{Authors information}

Affiliations: Key Laboratory of Cryptography of Zhejiang Province, Hangzhou Normal University, Hangzhou 311121, China

Contributions: Q.X. B.H. and K.L. have analyzed the security of Banerjee et al.'s scheme and have modeled and designed the protocol. K.L. and W.T. Wrote the paper. X.T. and L.H.Proved the authentication and security of the proposed scheme. B.H. and K.L.Verified the authentication, security and anonymity of the proposed scheme in the latest version 1.95 of ProVerif. Q.X. B.H. and K.L. revised the manuscript. All authors read and approved the final manuscript.

\section{Corresponding author:}

Correspondence to Qi XIE. 


\section{References}

1. W Fan, X Lili, K Saru, L Xiong. An improved and provably secure three-factor user authentication scheme for wireless sensor networks. P. P. Net. Appl. 11, 1-20(2018).

2. V R Maneesha, R Prabha, H Thirugnanam, R A Devidas, R K Pathinarupothi, Achieving sustainability through smart city applications: protocols, systems and solutions using I,oT and wireless sensor network. CSI. Tran. ICT. 8, 1-18(2020).

3. Q Xie, L Hwang, Security enhancement of an anonymous roaming authentication scheme with two-factor security in smart city. Neurocomputing, 347, 131-138(2019)

4. T Gaber, S Abdelwahab, M Elhoseny, A E Hassanien, Trust-based secure clustering in WSN-based intelligent transportation systems. Com. Net. 146, 151-158(2018)

5. R Watro, D Kong, S Cuti, C Gardiner, P Kruus, P K Tiny, securing sensor networks with public key technology. Proceedings of the 2nd ACM workshop on Security of ad hoc and sensor networks. ACM, 2004, pp.59-64.

6. M L Das, Two-factor user authentication in wireless sensor networks. IEEE transactions on wireless communications. 8(3),1086-1090(2009).

7. M K Khan, K Alghathbar, Cryptanalysis and security improvements of 'two-factor user authentication in wireless sensor networks'. Sensors. 10(3), 2450-2459(2010).

8. H L Yeh, T H Chen, P C Liu, T H Kim, H W Wei, A secured authentication protocol for wireless sensor networks using elliptic curves cryptography. Sensors. 11(5),4767-4779(2011).

9. K Xue, C Ma, P Hong, R Ding, A temporal-credential-based mutual authentication and key agreement scheme for wireless sensor networks. Journal of Network and Computer Applications. 36(1), 316-323(2013).

10. Q Jiang, S Zeadally, J Ma, D He, Lightweight three-factor authentication and key agreement protocol for Internet-integrated wireless sensor networks. IEEE Access. 5(1), 3376-3392(2017).

11. M Turkanović, B Brumen, M Hölbl, A novel user authentication and key agreement scheme for heterogeneous ad hoc wireless sensor networks, based on the Internet of Things notion. Ad Hoc Networks. 20, 96-112(2014).

12. S Banerjee, C Chunka, S Sen, R S Goswami, An Enhanced and Secure Biometric Based User Authentication Scheme in Wireless Sensor Networks Using Smart Cards. Wireless Personal Communications, 1-28(2019).

13. L Yanrong, L Lixiang, Y Xing, Y Yixian, Robust biometrics based authentication and key agreement scheme for multi-server environments using smart cards. PLoS One. 10(5), e0126323(2015).

14. T S Messerges, E A Dabbish, R H Sloan, Examining smart-card security under the threat of power analysis attacks. IEEE transactions on computers. 51(5), 541-552(2002). 
15. V S Miller, Use of elliptic curves in cryptography. Conference on the theory and application of cryptographic techniques. Springer, Berlin, Heidelberg.1985, pp.417-426

16. N Koblitz, Elliptic curve cryptosystems. Mathematics of computation. 48(177), 203-209(1987).

17. A K Das, A secure and effective biometric-based user authentication scheme for wireless sensor networks using smart card and fuzzy extractor. International Journal of Communication Systems. 30(1),1- 25(2017).

18. Y Dodis, L Reyzin, A Smith, Fuzzy extractors: How to generate strong keys from biometrics and other noisy data. International conference on the theory and applications of cryptographic techniques. (Springer, Berlin, Heidelberg, 2004, pp.523-540.

19. Ding W, Gu Q, H Cheng, W Ping, The request for better measurement: A comparative evaluation of two-factor authentication schemes. in Proc. 11th ACM Asia Conf. Comput. Commun. Secur., 475-486(2016)

20. B Blanchet, An Efficient Cryptographic Protocol Verifier Based on Prolog Rules. 14th IEEE Computer Security Foundations Workshop (CSFW-14), 2014, pp.82-96

21. Y Choi, Y Lee, D Won, Security improvement on biometric based authentication scheme for wireless sensor networks using fuzzy extraction. International Journal of Distributed Sensor Networks, 4,1-16(2016)

22. Y Park, Y Park, Three-Factor User Authentication and Key Agreement Using Elliptic Curve Cryptosystem in Wireless Sensor Networks. Sensors, 16(12), 2123(2016)

23. S Challa, M Wazid, A K Das, Kumar, N.; Goutham, R.A.; Yoon, E.J.; Yoo, K.Y. Secure Signature-Based Authenticated Key Establishment Scheme for Future IoT Applications. IEEE Access, 3028- 3043(2015)

24. $\mathrm{L} \mathrm{Xu}, \mathrm{F} \mathrm{Wu}$, Cryptanalysis and improvement of a user authentication scheme preserving uniqueness and anonymity for connected health care. Journal of medical systems, 39(10),(2015)

25. H H Kilinc, T Yanik, A survey of sip authentication and key agreement schemes. IEEE Communications Surveys and Tutorials, 16(2),1005-1023(2014)

\section{Figure Title and Legend Section:}

Fig 1: Simulation result of the authentication 
Query inj-event $(g w n f c c e p t U\langle U i\rangle\rangle=\Rightarrow$ inj-event $\langle$ martcardfccept $\langle U i\rangle\rangle$ Completing. . .

ok, secrecy assumption verified: fact unreachable attacker(GWNPS[])

ok, secrecy assumption verified: fact unreachable attacker $\langle\mathrm{Sg}[\mathrm{J}\rangle$

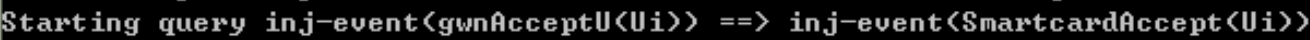

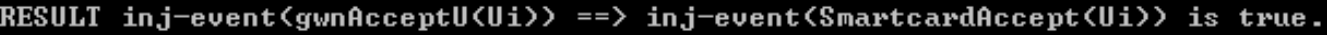

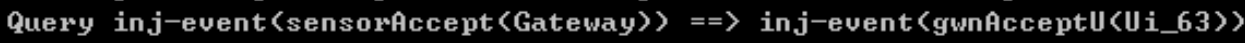

Completing ... .

ok, secrecy assumption verified: fact unreachable attacker (GWNPSj[])

ok, secrecy assumption verified: fact unreachable attacker $\langle\mathrm{Sg}[\mathrm{C}\rangle$

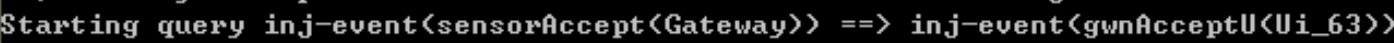

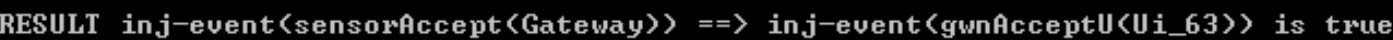

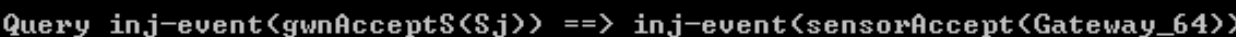

Completing...

ok, secrecy assumption verified: fact unreachable attacker (GWNPSj[])

ok, secrecy assumption verified: fact unreachable attacker $\langle\mathrm{Sg}[\mathrm{J}\rangle$

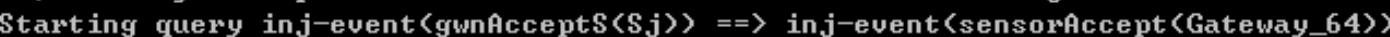

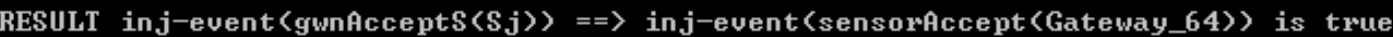

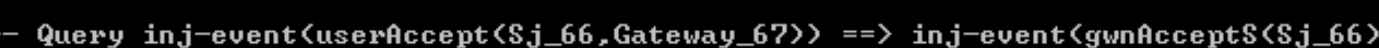

Completing... -

ok, secrecy assumption verified: fact unreachable attacker (GWNPSj[])

ok, secrecy assumption verified: fact unreachable attacker $\langle\mathrm{Sg}[\mathrm{J}\rangle$

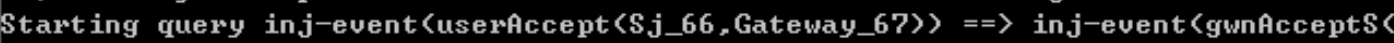
Sj_66)>

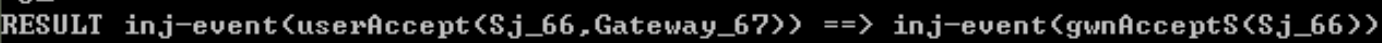

is true.

Fig 2: Simulation result of the session key

Query not attacker〈SKsu[]〉

Completing...

ok, secrecy assumption verified: fact unreachable attacker (GWNPSj[]>

ok, secrecy assumption verified: fact unreachable attacker $\langle\mathrm{Sg}[\mathrm{]}\rangle$

Starting query not attacker〈SKsu[])

RESULT not attacker 〈SKsu[]〉 is true.

Query not attacker〈SKus []〉

Completing...

ok, secrecy assumption verified: fact unreachable attacker 〈GWNPSj[]〉

ok, secrecy assumption verified: fact unreachable attacker $\langle\mathrm{Sg}[]\rangle$

starting query not attacker (Skus[])

RESULT not attacker 〈Skus []〉 is true. 


\section{Figures}

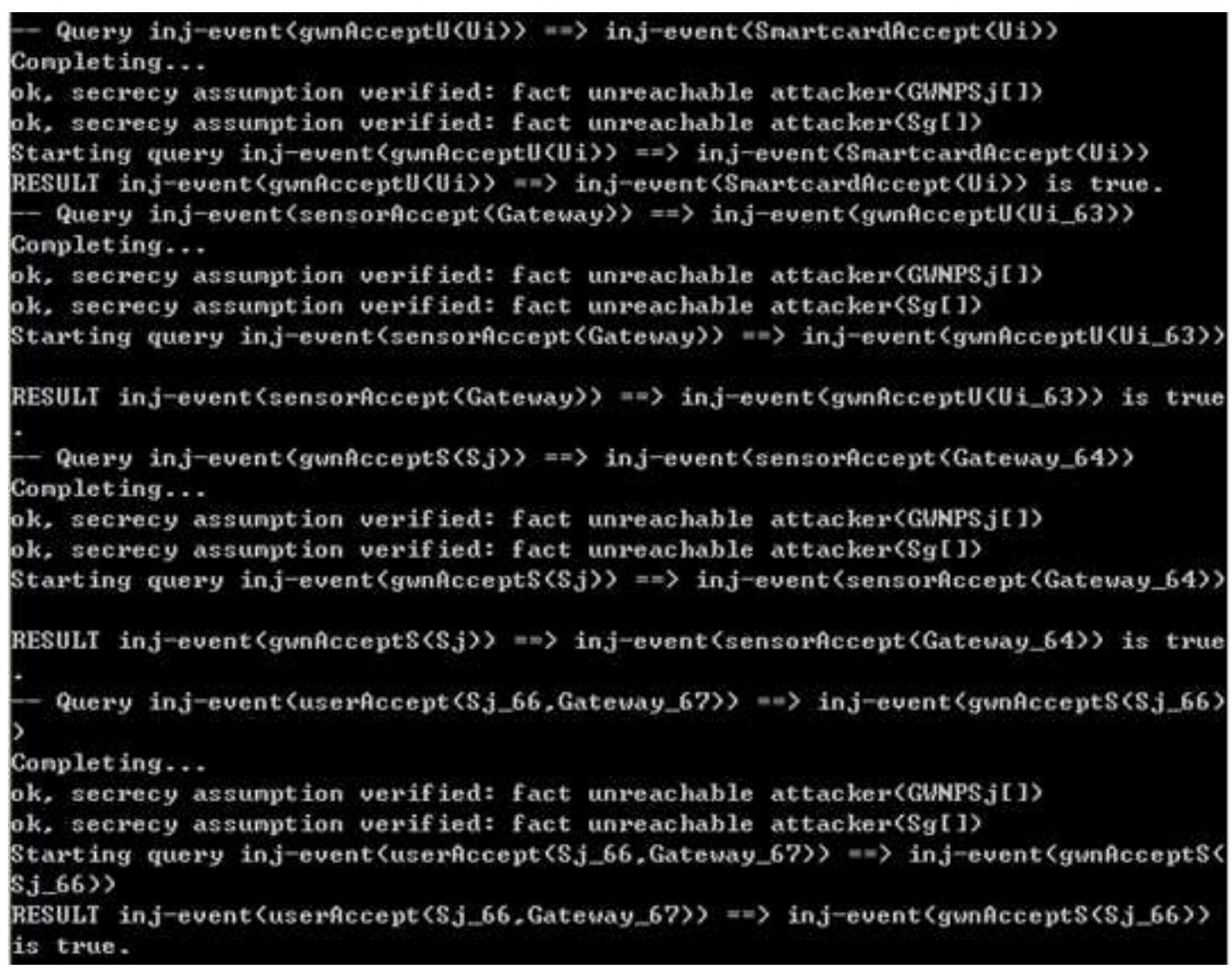

\section{Figure 1}

Simulation result of the authentication

Query not attacker(SKsu[])

Conpleting...

ok. secrecy assumption verified: fact unreachable attacker (GUNPSj[])

ok, secrecy assumption verified: fact unreachable attacker'(Sg[1)

Starting query not attacker(S)ksu[j])

RESULT not attacker (SKsu[ ] is true.

-- Query not attacker(SKus [])

Completing...

ok, secrecy assumption verif ied: fact unreachable attacker-(GUNPSj[])

ok. secrecy assunption verified: fact unreachable attacker(Sg[])

Starting query not attacker(sKus [])

RESULT not attacker $\langle S K u s[]\rangle$, is true.

\section{Figure 2}

Simulation result of the session key 